\title{
On the Converse of Caristi's Fixed Point Theorem
}

\author{
by \\ Szymon GEĄB \\ Presented by Czestaw BESSAGA
}

Summary. Let $X$ be a nonempty set of cardinality at most $2^{\aleph_{0}}$ and $T$ be a selfmap of $X$. Our main theorem says that if each periodic point of $T$ is a fixed point under $T$, and $T$ has a fixed point, then there exist a metric $d$ on $X$ and a lower semicontinuous map $\phi: X \rightarrow \mathbb{R}_{+}$such that $d(x, T x) \leq \phi(x)-\phi(T x)$ for all $x \in X$, and $(X, d)$ is separable. Assuming $\mathrm{CH}$ (the Continuum Hypothesis), we deduce that $(X, d)$ is compact.

We use the notation $\mathbb{R}_{+}=[0, \infty), \mathbb{N}=\{0,1,2, \ldots\}$ and $\mathbb{Z}=\{ \pm k: k \in \mathbb{N}\}$. Let $X$ be a nonempty set and let $T: X \rightarrow X$ be a selfmap. By Fix $T$ and Per $T$ we denote the sets of all fixed and periodic points of $T$, respectively (i.e., Fix $T=\{x \in X: T x=x\}$ and $\operatorname{Per} T=\left\{x \in X: T^{n} x=x\right.$ for some $n \in \mathbb{N} \backslash\{0\}\}$ ). If Fix $T=\operatorname{Per} T \neq \emptyset$, the abstract dynamical system $(X, T)$ is called a C-system. If $(X, T)$ is a C-system and Fix $T$ is a singleton, then it is called a $B$-system. The following theorems show connections between B-systems and Banach's fixed point theorem, and between C-systems and Caristi's [2] fixed point theorem.

Theorem 1 (Bessaga [1]). Let $(X, T)$ be a B-system and $\alpha \in(0,1)$. Then there exists a complete metric $d$ on $X$ such that

$$
\forall x, y \in X \quad d(T x, T y) \leq \alpha d(x, y) .
$$

TheOREM 2 (Janoš [5]). Let $(X, T)$ be a B-system of cardinality at most $2^{\aleph_{0}}$ and $\alpha \in(0,1)$. Then there exists a separable metric $d$ on $X$ such that

$$
\forall x, y \in X \quad d(T x, T y) \leq \alpha d(x, y) .
$$

Theorem 3 (Jachymski [4]). Let $(X, T)$ be a C-system. Then there exist a complete metric $d$ on $X$ and a lower semicontinuous function $\phi: X \rightarrow \mathbb{R}_{+}$

2000 Mathematics Subject Classification: Primary 47H10, 54H25; Secondary 03E50.

Key words and phrases: abstract dynamical system, fixed point, periodic point, Continuum Hypothesis. 
such that

$$
\forall x \in X \quad d(x, T x) \leq \phi(x)-\phi(T x)
$$

Every $\alpha$-contraction $T$ satisfies (1) for $\phi(x)=\frac{d(x, T x)}{1-\alpha}$ (for details see $[3$, p. 16]). Since there exist discontinuous mappings satisfying (1), the family of all mappings for which (1) holds is essentially greater than the family of all contractions. The next example shows that in $\mathbb{R}$ these two families have even different cardinalities.

ExAmple 1 . Let $B$ be a subset of $\mathbb{R}$ such that $0 \in B$. Consider the map $T_{B}: \mathbb{R} \rightarrow \mathbb{R}$ given by

$$
T_{B}(x)= \begin{cases}x & \text { if } x \notin B, \\ 0 & \text { if } x \in B,\end{cases}
$$

and let $\phi(x)=|x|, x \in \mathbb{R}$. Then

$$
\forall x \in X \quad d\left(x, T_{B} x\right) \leq \phi(x)-\phi\left(T_{B} x\right) .
$$

We have $T_{B}^{-1}(0)=B$. Since $B$ can be an arbitrary subset of the real line, assuming that $B$ is non-Borel (nonmeasurable, without the Baire property, etc.) we infer that $T_{B}$ is non-Borel (nonmeasurable, without the Baire property, etc.). This also shows that there are $2^{2^{\aleph_{0}}}$ maps $T_{B}$, but only $2^{\aleph_{0}}$ contractions of $\mathbb{R}$.

Janoš [6, Theorem 4.6] proved that there exists a B-system $(X, T)$ of cardinality $2^{\aleph_{0}}$ such that there is no metric $d$ on $X$ which is complete separable and such that $T$ is a Banach contraction relative to $d$. The proof is nonconstructive and based on comparison of cardinalities. We propose two constructive examples. Example 2 is simple but it assumes $\neg \mathrm{CH}$. Example 3 gives a more involved construction in $\mathrm{ZFC}$.

ExAmple 2. Assume $\neg$ CH. Let $X=2^{\aleph_{0}}$ and let $T(\alpha)$ equal $\omega_{1}$ if $\alpha \leq \omega_{1}$, and 0 otherwise. Obviously $(X, T)$ is a B-system. Since $T^{-1}\left(\left\{\omega_{1}\right\}\right)$ is of cardinality $\omega_{1}<2^{\aleph_{0}}$, there cannot exist a complete separable metric $d$ on $X$ such that $T$ is a Banach contraction relative to $d$ (see [7, Theorem 3.2.7]).

ExAmPlE 3. Here we use the notation from [7]. Fix $\mathcal{A} \subset \mathcal{P}(\mathbb{N})$ of cardinality $2^{\aleph_{0}}$. Enumerate sets in $\mathcal{A}$ as $\left\{A_{\alpha}: \alpha<2^{\aleph_{0}}\right\}$. Put $X=A \cup B \cup\{0\}$, where

$$
\begin{aligned}
& A=\bigcup_{\alpha<2^{\aleph_{0}}}\left((\mathbb{N} \times\{0\} \times\{\alpha\}) \cup\left(A_{\alpha} \times\{1\} \times\{\alpha\}\right)\right), \\
& B=\left\{(0, \alpha): \alpha<2^{\aleph_{0}}\right\} .
\end{aligned}
$$

Define $T: X \rightarrow X$ by $T(n, i, \alpha)=(n-1,0, \alpha)$ for $n>0, T(0, i, \alpha)=(0, \alpha)$, $T(0, \alpha)=0$ and $T(0)=0$. Then $(X, T)$ is a B-system.

Suppose there exists a metric $d$ on $X$ such that $(X, d)$ is a complete separable metric space and $T$ is a Banach contraction. Since $X$ is a Polish 
space, so is its closed subset $B \cup\{0\}=T^{-1}(\{0\})$. Recall that $\mathcal{P}(\mathbb{N})$ can be treated as the Cantor space $\mathcal{C}=\{0,1\}^{\mathbb{N}}$.

We have

$$
H \in \mathcal{A} \Leftrightarrow \exists x \in B\left(H=\left\{n: \exists y, z \in X\left(y \neq z, T^{n+1} y=T^{n+1} z=x\right)\right\}\right) .
$$

Hence $\mathcal{A}$ is the projection into $\mathcal{P}(\mathbb{N})$ of the set

$$
\begin{aligned}
&\left\{(H, x) \in \mathcal{P}(\mathbb{N}) \times B: H=\left\{n: \exists y, z \in X\left(y \neq z, T^{n+1} y=T^{n+1} z=x\right)\right\}\right\} \\
&=\left\{(H, x): \forall n \in \mathbb{N}\left(n \in H \Leftrightarrow \exists y, z \in X\left(y \neq z, T^{n+1} y=T^{n+1} z=x\right)\right)\right\} \\
&= \bigcap_{n \in \mathbb{N}}\left[\left(\{(H, x): n \in H\} \cup\left\{(H, x): \forall y, z \in X \neg\left(y \neq z, T^{n+1} y=T^{n+1} z=x\right)\right\}\right)\right. \\
&\left.\cap\left(\{(H, x): n \notin H\} \cup\left\{(H, x): \exists y, z \in X\left(y \neq z, T^{n+1} y=T^{n+1} z=x\right)\right\}\right)\right] .
\end{aligned}
$$

Notice that the sets $\{(H, x): n \notin H\}$ and $\{(H, x): n \in H\}$ are clopen for all $n \in \mathbb{N}$. Since $\left\{(H, x, y, z) \in \mathcal{P}(\mathbb{N}) \times B \times X^{2}: \neg\left(y \neq z\right.\right.$ and $T^{n+1} y=$ $\left.\left.T^{n+1} z=x\right)\right\}$ is Borel, the set $\left\{(H, x): \forall y, z \in X \neg\left(y \neq z, T^{n+1} y=\right.\right.$ $\left.\left.T^{n+1} z=x\right)\right\}$ is coanalytic and $\left\{(H, x): \exists y, z \in X\left(y \neq z, T^{n+1} y=\right.\right.$ $\left.\left.T^{n+1} z=x\right)\right\}$ is analytic. Hence

$$
\left\{(H, x) \in \mathcal{P}(\mathbb{N}) \times B: H=\left\{n: \exists y, z \in X\left(y \neq z, T^{n+1} y=T^{n+1} z=x\right)\right\}\right\}
$$

is in the projective class $\Sigma_{2}^{1}(\mathcal{P}(\mathbb{N}) \times B)$. Since $\Sigma_{2}^{1}$ is closed under projections, $\mathcal{A}$ is in $\Sigma_{2}^{1}(\mathcal{P}(\mathbb{N}))$.

Consider a $\Pi_{2}^{1}$ set in $\mathcal{P}(\mathbb{N})$ which is not $\Sigma_{2}^{1}$. (Some natural examples of such sets are known. In [7, Exercise 37.15], an example of a $\Sigma_{2}^{1}$-complete subset of the hyperspace $\mathcal{K}(\mathcal{C})$ of the Cantor space $\mathcal{C}$ is given. Its complement is $\Pi_{2}^{1}$-complete, and it can be viewed as a subset of $\mathcal{P}(\mathbb{N})=\mathcal{C}$, since $\mathcal{K}(\mathcal{C})$ is homeomorphic to $\mathcal{C}$.) Then we obtain a B-system $(X, T)$ for which there is no complete and separable metric $d$ on $X$ such that $T$ is a contraction.

By $O_{T}(x)$ we denote the orbit of the point $x$ in the sense of Kuratowski (i.e., $\left.O_{T}(x)=\left\{y \in X: \exists m, n \in \mathbb{N}\left(T^{m} x=T^{n} y\right)\right\}\right)$. A metric space is called precompact if its completion is compact. Since a precompact space is a subspace of compact space, it is separable.

Now, we shall prove our main result which is an analogue of Theorem 2 for C-systems.

TheOREM 4. Let $(X, T)$ be a C-system of cardinality at most $2^{\aleph_{0}}$. There exist a precompact metric $d$ on $X$ and a lower semicontinuous function $\phi: X \rightarrow \mathbb{R}_{+}$(relative to $d$ ) such that

$$
\forall x \in X \quad d(x, T x) \leq \phi(x)-\phi(T x) .
$$

Proof. Fix $x_{0} \in$ Fix $T$. Put $\mathcal{A}=\left\{O_{T}(x): x \in X\right\}$. Let $A \subset X$ be a selector of $\mathcal{A}$, i.e. for all $x \in X$ the set $A \cap O_{T}(x)$ is a singleton. Let $x \in X \backslash\left\{x_{0}\right\}$. Pick $a \in A$ such that $x \in O_{T}(a)$. Let $m=\min \{k \in \mathbb{N}: \exists n \in \mathbb{N}$ 
$\left.\left(T^{n} x=T^{k} a\right)\right\}$ and $n=\min \left\{k \in \mathbb{N}:\left(T^{k} x=T^{m} a\right)\right\}$. Put $h(x)=n-m$, and $h\left(x_{0}\right)=-\infty$.

Since Fix $T=\operatorname{Per} T$, the map $h: X \rightarrow \mathbb{Z} \cup\{-\infty\}$ has the following property:

$$
\forall x \in X \quad(x \neq T x \Rightarrow h(x)>h(T x)) .
$$

Let $g_{k}: h^{-1}(\{k\}) \rightarrow\left[\pi / 2^{|k|}-\pi / 2^{|k|+2}, \pi / 2^{|k|}+\pi / 2^{|k|+2}\right]$ be a one-to-one map, for every $k \in \mathbb{Z}$. Define $G: X \rightarrow \mathbb{C}$ by

$$
G(x)= \begin{cases}\exp \left(-i g_{k}(x)\right) & \text { if } x \in h^{-1}(\{k\}) \text { and } k<0 \\ \exp \left(i g_{k}(x)\right) & \text { if } x \in h^{-1}(\{k\}) \text { and } k \geq 0 \\ 1 & \text { if } h(x)=-\infty\end{cases}
$$

Next define $\phi: X \rightarrow \mathbb{R}$ by

$$
\phi(x)= \begin{cases}(4+k) \pi & \text { if } x \in h^{-1}(\{k\}) \text { and } k>0, \\ 4 \pi / 2^{|k|} & \text { if } x \in h^{-1}(\{k\}) \text { and } k \leq 0, \\ 0 & \text { if } x=x_{0} .\end{cases}
$$

Since $G: X \rightarrow \mathbb{C}$ is one-to-one, we may consider $X$ as a subspace of $\{\exp (i x): x \in[-\pi, \pi]\}$ with a metric given by the length of the shorter arc joining two points.

Let $x \in X$. We shall show that (1) holds.

CASE 1: $x=x_{0}$. Then $T x_{0}=x_{0}$ and (1) obviously holds.

CASE $2: k=h(x) \leq 0$ and $x \neq x_{0}$. Then $d(x, T x) \leq \pi / 2^{|k|}+\pi / 2^{|k|+2}$ and $\phi(x)-\phi(T x)=4 \pi / 2^{|k|}-4 \pi / 2^{|k|+1}=4 \pi / 2^{|k|+1}=\pi / 2^{|k|-1}$. Hence (1) holds.

CASE 3: $h(x) \geq 1$. Then $d(x, T x) \leq \pi, \phi(x)-\phi(T x)=\pi$ and (1) holds.

We now show that $\phi$ is lower semicontinuous. Let $\left\{x_{n}\right\}_{n \in \mathbb{N}}$ be a sequence of elements of $X$ convergent to $y \in X$.

CAse 1: $y=x_{0}$. Then $\phi\left(x_{n}\right) \geq 0$ for all $n \in \mathbb{N}$, and $\phi(y)=0$. Hence $\phi(y) \leq \liminf \phi\left(x_{n}\right)$.

CASE 2: $y \neq x_{0}$. Then there exists $n_{0}$ such that $\phi(y)=\phi\left(x_{m}\right)$ for all $m \geq n_{0}$. Hence $\phi(y)=\lim \phi\left(x_{n}\right)$.

Under $\mathrm{CH}$ we can improve the assertion of Theorem 4.

Corollary 5. (CH) Let $(X, T)$ be a C-system of cardinality at most $2^{\aleph_{0}}$. There exist a metric $d$ on $X$ and a lower semicontinuous function $\phi$ : $X \rightarrow \mathbb{R}_{+}$(relative to this metric) such that

$$
\forall x \in X \quad d(x, T x) \leq \phi(x)-\phi(T x),
$$

and $(X, d)$ is compact and zero-dimensional. 
Proof. Since (under $\mathrm{CH}$ ) the sets $h^{-1}(\{k\})$ are countable or of cardinality $2^{\aleph_{0}}$, we may assume that for all $k \in \mathbb{Z}, g_{k}$ is a one-to-one function whose range is a countable compact or Cantor-like subset of $\left[-\pi / 2^{|k|+1}, \pi / 2^{|k|+1}\right]$. The result follows.

EXAMPLE 4. Corollary 5 cannot be improved to get a function $\phi$ which is continuous even if $(X, T)$ is a B-system. To see this let $X=\mathbb{N}^{\mathbb{N}}$ and let $\alpha \in X$. If $\alpha=(0,0,0, \ldots)$, then put $T(\alpha)=\alpha$. If $\alpha \neq(0,0,0, \ldots)$, then put $T(\alpha)=(\alpha(0), \alpha(1), \ldots, \alpha(n-1), \alpha(n)-1, \alpha(n+1), \ldots)$, where $n=\min \{k \in \mathbb{N}: \alpha(k)>0\}$. Then $(X, T)$ is a B-system. Suppose that there exist a compact metric $d$ on $X$ and a continuous function $\phi: X \rightarrow \mathbb{R}_{+}$such that

$$
\forall x \in X \quad d(x, T x) \leq \phi(x)-\phi(T x) .
$$

We shall define inductively a sequence $\alpha_{\xi}, \xi<\omega_{1}$. Let $\alpha_{0}=(0,0,0, \ldots)$. Assume that $0<\gamma<\omega_{1}$ and that we have defined $\alpha_{\xi}$ for all $\xi<\gamma<\omega_{1}$, with $\phi\left(\alpha_{\xi}\right)<\phi\left(\alpha_{\eta}\right)$ for all $\xi, \eta$ such that $\xi<\eta<\gamma$. If $\gamma=\eta+1$ and $\alpha_{\eta}=$ $\left(n_{0}, n_{1}, n_{2}, \ldots\right)$ then put $\alpha_{\gamma}=\left(n_{0}+1, n_{1}, n_{2}, \ldots\right)$. Since $T \alpha_{\gamma}=T \alpha_{\eta}$, we have $\phi\left(\alpha_{\eta}\right)<\phi\left(\alpha_{\gamma}\right)$. If $\gamma$ is a limit ordinal, pick an increasing sequence $\left\{\eta_{n}\right\}_{n \in \mathbb{N}}$ of ordinals less than $\gamma$, with $\bigcup\left\{\eta_{n}: n \in \mathbb{N}\right\}=\gamma$. By the compactness of $X$ we choose a convergent subsequence $\left\{\alpha_{\eta_{n_{k}}}\right\}_{k \in \mathbb{N}}$ of $\left\{\alpha_{\eta_{n}}\right\}_{n \in \mathbb{N}}$. Let $\alpha_{\gamma}=$ $\lim _{k \rightarrow \infty} \alpha_{\eta_{n_{k}}}$. By inductive assumption and continuity of $\phi$ we easily see that $\phi\left(\alpha_{\eta}\right)<\phi\left(\alpha_{\gamma}\right)$ for all $\eta<\gamma$. Thus by transfinite induction we have defined an uncountable and strictly increasing sequence $\left\{\phi\left(\alpha_{\gamma}\right): \gamma<\omega_{1}\right\}$ in $\mathbb{R}$, which yields a contradiction.

The following example shows that Theorem 2 cannot be improved to get the metric $d$ precompact.

ExAmple 5. Let $(X, T)$ be a B-system such that $T$ is a surjection. Suppose that there is a bounded metric $d$ making $T$ an $\alpha$-contraction. Then $\operatorname{diam} T^{n}(X) \leq \alpha^{n} \operatorname{diam} X$. Hence $\bigcap T^{n}(X)$ is a singleton, but since $T$ is a surjection we have $\bigcap T^{n}(X)=X$, which is true only when $X$ is singleton. Since a precompact metric is bounded, $d$ cannot be precompact.

Now, we give variants of Theorem 4 and Corollary 5 with slightly modified assertions.

TheOREM 6. Let $(X, T)$ be a C-system of cardinality at most $2^{\aleph_{0}}$. There exist a separable metric $d$ on $X$ and a continuous function $\phi: X \rightarrow \mathbb{R}_{+}$ (relative to d) such that

$$
\forall x \in X \quad d(x, T x) \leq \phi(x)-\phi(T x) .
$$

Proof. Let $h: X \rightarrow \mathbb{Z} \cup\{-\infty\}$ be a function defined as in Theorem 4 . Let $g_{k}: h^{-1}(\{k\}) \rightarrow\left[2^{-k}-2^{-k-2}, 2^{-k}+2^{-k-2}\right]$ be a one-to-one map. Define 
$G: X \rightarrow \mathbb{R}$ by

$$
G(x)= \begin{cases}g_{k}(x) & \text { if } x \in h^{-1}(\{k\}), \\ 0 & \text { if } h(x)=-\infty\end{cases}
$$

and $\phi: X \rightarrow \mathbb{R}_{+}$by

$$
\phi(x)= \begin{cases}2^{-k} & \text { if } x \in h^{-1}(\{k\}), \\ 0 & \text { if } h(x)=-\infty .\end{cases}
$$

Since $G$ is one-to-one, we may consider $X$ as a subspace of $\mathbb{R}$ with the Euclidean metric.

Corollary 7. (CH) Let $(X, T)$ be a C-system of cardinality at most $2^{\aleph_{0}}$. There exist a metric $d$ on $X$ and a continuous function $\phi: X \rightarrow \mathbb{R}_{+}$ (relative to this metric) such that

$$
\forall x \in X \quad d(x, T x) \leq \phi(x)-\phi(T x),
$$

and $(X, d)$ is complete and zero-dimensional.

Acknowledgements. I would like to thank Jacek Jachymski for his inspiration. I am grateful to Adam Krawczyk who has suggested to me an idea used in Example 3.

\section{References}

[1] C. Bessaga, On the converse of the Banach fixed point principle, Colloq. Math. 7 (1959), 41-43.

[2] J. Caristi, Fixed point theorems for mappings satisfying inwardness conditions, Trans. Amer. Math. Soc. 215 (1976), 241-251.

[3] J. Dugundji and A. Granas, Fixed Point Theory I, Polish Sci. Publ., Warszawa, 1982.

[4] J. Jachymski, Converses to fixed point theorems of Zermelo and Caristi, Nonlinear Anal. 52 (2003), 1455-1463.

[5] L. Janoš, An application of combinatorial techniques to a topological problem, Bull. Austral. Math. Soc. 9 (1973), 439-443.

[6] -, Compactification and linearization of abstract dynamical systems, Acta Univ. Carolin. Math. Phys. 38 (1997), 63-70.

[7] A. S. Kechris, Classical Descriptive Set Theory, Springer, New York, 1998.

Szymon Głąb

Institute of Mathematics

Technical University of Łódź

Al. Politechniki 11

90-924 Łódź, Poland

E-mail: szymon_glab@yahoo.com
Institute of Mathematics Polish Academy of Sciences Śniadeckich 8 00-956 Warszawa, Poland

Received March 20, 2004;

received in final form September 30, 2004 